\title{
Prefontal cortex in long-term memory: an "interference" approach using magnetic stimulation
}

Simone Rossi, Stefano F. Cappa, Claudio Babiloni, Patrizio Pasqualetti, Carlo Miniussi, Filippo Carducci, Fabio Babiloni and Paolo M. Rossini

Nat. Neurosci. 4, 948-952 (2001)

The title of this article contained a typographical error. It should have read as follows:

\section{Prefrontal cortex in long-term memory: an "interference" approach using magnetic stimulation}

\section{Ion channel properties underlying axonal action potential initiation in pyramidal neurons}

Costa M. Colbert and Enhui Pan

Nat. Neurosci. 5, 533-538 (2002)

A printer's error introduced an extraneous diagonal line into Fig. $2 \mathrm{~b}$ on page 534 . The correct figure is reproduced below.

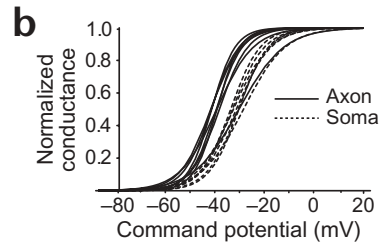

Fig. 2. $\mathrm{Na}^{+}$channel properties differ between
the soma and axon. (b) Voltage dependence of
activation in somatic and axonal patches. Each
curve is the best-fit Boltzmann for an individual
patch. Axonal $\mathrm{Na}^{+}$channels (solid lines) were
activated by less depolarization than somatic
channels (dotted lines).

corrigenda

\section{Neurotrophins use the Erk5 pathway to mediate a retrograde survival response}

Fiona L. Watson, Heather M. Heerssen, Anita Bhattacharyya, Laura Klesse, Michael Z. Lin and Rosalind A. Segal

Nat. Neurosci. 4, 981-988 (2001)

In Fig. 5e on page 986, the pluses and minuses for lines "PD to DA" and "PD to CB" were incorrect. The conclusions stated in the text and the experimental description in the figure legend were correct. The corrected figure is reproduced below.

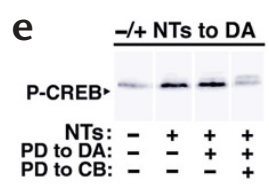

\begin{abstract}
Fig. 5. Activation of Erk5 promotes survival. (e) Neurons in compartmented cultures were treated with PD98059 (PD) to distal axons or cell bodies, as indicated. Distal axons were stimulated with neurotrophins and cell body lysates were immunoblotted for P-CREB. PD treatment of distal axons alone does not prevent CREB phosphorylation. When PD is applied to the cell bodies, CREB phosphorylation is inhibited.
\end{abstract}

\section{A-kinase anchoring proteins in amygdala are involved in auditory fear memory}

Marta A.P. Moita, Raphael Lamprecht, Karim Nader and Joseph E. LeDoux

Nat. Neurosci. 5, 837-838 (2002)

The authors wish to correct their supplementary methods online, which gave the wrong sources for three antibodies. The mouse anti-RII $\alpha$ and anti-RII $\beta$ antibodies were obtained from Transduction Laboratories (San Diego, California), and the rabbit antiAKAP150 antibody was obtained from Santa Cruz Biotechnology (Santa Cruz, California). 